\title{
Artículos
}

\section{Propuesta de una taxonomía de los centros de interés en los estudios de disponibilidad léxica}

Proposta de uma taxonomia dos centros de interesse nos estudos de disponibilidade lexical Proposal for a taxonomy of centers of interest in lexical availability studies

\author{
Inmaculada Clotilde Santos Díaz \\ Ester Trigo lbáñez ${ }^{2}$ \\ Manuel Francisco Romero Oliva ${ }^{3}$
}

\section{RESUMEN}

Los estudios de disponibilidad léxica recopilan, con fines didácticos, unidades léxicas en centros de interés. Se ha destacado el diferente comportamiento de los centros de interés partiendo del número de unidades. Además, se han aportado clasificaciones según otros parámetros relacionados con la estructura interna. Sin embargo, no existe ninguna clasificación aplicada a más de un idioma. Pretendemos proponer una taxonomía a partir de la creación de un índice estructural de los centros

1. Universidad de Málaga. Málaga - Espanha. https://orcid.org/0000-0002-0066-7783. E-mail: santosdiaz@uma.es.

2. Universidad de Cádiz. Cádiz - Espanha. http://orcid.org/0000-0003-3035-4398. Email: ester.trigo@uca.es.

3. Universidad de Cádiz. Cádiz-Espanha. http://orcid.org/0000-0002-6854-0682. E-mail: manuelfrancisco.romero@uca.es. 
de interés. Para ello, demostraremos el diferente comportamiento de los centros de interés tanto en lengua materna (español) como en lengua extranjera (inglés y francés) y estableceremos una medida de valoración de sus características.

Palabras clave: disponibilidad léxica; psicolingüistica; indice estructural; enseñanza de lenguas.

\title{
RESUMO
}

Os estudos de disponibilidade lexical recolhem, para fins didáticos, unidades lexicais em centros de interesse (CI). Foi salientado o comportamento diferente dos CI com base no número de unidades. Além disso, as classificações foram fornecidas de acordo com outros parâmetros relacionados com a estrutura interna. No entanto, não existe uma classificação aplicada a mais do que uma língua. Pretendemos propor uma taxonomia baseada na criação de um índice estrutural de CI. Para o efeito, demonstraremos os diferentes comportamentos dos centros de interesse tanto na língua materna (espanhol) como na lingua estrangeira (inglês e francês) e estabeleceremos uma medida para avaliar as suas características.

Palavras-chave: disponibilidade lexical; psicolinguística; índice estrutural; ensino de línguas.

\begin{abstract}
Lexical availability studies collect, for teaching purposes, lexical units in centers of interest. The different behavior of centers of interest has been highlighted based on the number of units. In addition, classifications have been provided according to other parameters related to the internal structure. However, there is no classification applied to more than one language. We intend to propose a taxonomy based on the creation of a structural index of centers of interest. To do this, we will demonstrate the different behavior of the centers of interest both in the mother tongue (Spanish) and in the foreign language (English and French) and we will establish an assessment measure of their characteristics.
\end{abstract}

Keywords: lexical abailability; psicolinguistics; structural index; language teaching. 


\section{Introducción}

Los estudios de disponibilidad léxica se inician en Francia a mediados del siglo pasado (Gougenheim, Michéa, Rivenc y Sauvageot 1956). Su primera aplicación fue didáctica, pues se pretendía compilar un vocabulario válido para enseñar francés a los habitantes de la antigua unión francesa en Canadá. Sin embargo, ha sido en el mundo hispánico donde estas investigaciones han encontrado su mayor ámbito de desarrollo. Esta proliferación ha sido posible, en gran parte, gracias a la existencia de un Proyecto Panhispánico de Disponibilidad Léxica (PPHDL), coordinado por el profesor Humberto López Morales, cuestión que ha permitido aunar criterios metodológicos y, en consecuencia, trazar una senda de investigación sólida y científicamente madura (Paredes 2012; López González 2014a).

Si nos centramos en un hablante concreto, "cuando hablamos de léxico disponible estamos haciendo referencia a un conjunto de unidades léxicas que están altamente disponibles para un hablante de una lengua concreta cuando se le pregunta por una determinada categoría semántica" (Hernández Muñoz y Tomé 2017: 100). Sin embargo, si queremos obtener el léxico disponible de un colectivo -o comunidad de habla- será preciso aplicar una prueba asociativa de fluidez categorial -oral o escrita- en la que los informantes deberán evocar todo el léxico posible en un tiempo concreto. Estas categorías -o áreas temáticas- son conocidas en el ámbito de la disponibilidad léxica como centros de interés ${ }^{4}$. Estos, aunque han permitido realizar interesantes comparaciones diatópicas y plantear estrategias para la selección del léxico en la enseñanza de lenguas, como detallan Samper Padilla, Bellón y Samper Hernández (2003), presentan diferentes características internas y niveles de productividad diversos. Además, con el avance de estos estudios no faltan las voces que abogan por una revisión de estos centros de interés (Paredes 2014; Tomé 2015;

4. Las investigaciones adscritas al PPHDL utilizan, como mínimo, los 16 centros de interés tradicionales: (01) Partes del cuerpo, (02) La ropa, (03) Partes de la casa (sin los muebles), (04) Los muebles de la casa, (05) Alimentos y bebidas, (06) Objetos colocados en la mesa para la comida, (07) La cocina y sus utensilios, (08) La escuela: muebles y materiales, (09) Iluminación, calefacción y medios para airear un recinto, (10) La ciudad, (11) El campo, (12) Medios de transporte, (13) Trabajos del campo y del jardín, (14) Los animales, (15) Juegos y distracciones, (16) Profesiones y oficios. 
Sánchez-Saus 2016, 2019; Hidalgo 2017) o que proponen centros de interés nuevos, como Castillo Fadić y Pino Castillo (2020) con La diabetes.

De acuerdo con estos planteamientos, de la cuantificación de la disponibilidad léxica en los centros de interés se desprende una diferente estructura según su productividad, heterogeneidad y densidad. Gougenheim, Michéa, Rivenc y Sauvageot (1964: 189-190) clasifican los centros por orden de dispersión creciente, es decir, según el número de vocablos aportados en cada centro de interés en los diferentes departamentos. Los investigadores franceses distinguen entre dos tipos de centros: aquellos que no llegan a los 400 vocablos y los que superan este umbral. Asimismo, asocian el grado de disponibilidad (basado en la frecuencia) de la palabra más "disponible" con la dispersión o número de vocablos de forma que en los centros con más de 400 palabras diferentes el grado de disponibilidad es menor debido a la falta de homogeneidad. Concluyen otorgando mayor relevancia para el estudio a los centros que pertenecen a la primera categoría, en los que se encuentran los centros denominados Les vetêments y Les parties $d u \operatorname{corps}^{6}$ y que serán objeto de análisis ulteriores.

Desde que Gougenheim et al. $(1956,1964)$ destacaron la similitud entre el comportamiento de los centros de interés de las cuatro encuestas llevadas a cabo y los clasificaron según la homogeneidad, es frecuente encontrar en los estudios sobre léxico disponible un apartado de análisis comparativos según el número de vocablos y palabras (Ávila y Villena 2010; Bellón 2011; Trigo y Romero 2011; Sánchez-Saus 2016; entre otros). Esos dos indicadores sirven como punto de partida para analizar la diferente ontogenia de los centros a través del índice de cohesión y el índice de densidad léxica.

El índice de cohesión, propuesto por Echeverría, Herrera, Moreno y Pradenas (1987: 68), "mide el grado de coincidencia en las respuestas de los sujetos para un mismo centro". Echeverría et al. (1987) analizaron la disponibilidad léxica de 200 estudiantes de primer curso y 200 de

5. Aquella que se encuentra con más frecuencia en los listados.

6. Con objeto de que los valores mayores indiquen más homogeneidad y prototipicidad se ha restado uno de forma que los centros con un índice más elevado sean los que dispongan de relaciones más fuertes. 
tercer curso de Educación Media de Concepción y aplicaron el cálculo del índice de cohesión. Dividieron los centros de interés según ese grado de cohesión semántica, distinguiendo entre centros de alto grado: 1) Cuerpo humano, 2) Medios de transporte, 3) La ropa, 4) Muebles, 5) Herramientas y materiales de construcción, 6) Alimentos, 7) Animales; y centros de bajo grado 8) Naturaleza, 9) La casa y 10) La cocina. Posteriormente, Echeverría (1991) explica cómo aplicar este índice y cómo interpretarlo.

El índice de densidad léxica fue empleado por primera vez por Alba (1995) en un estudio sobre disponibilidad léxica de una muestra de 347 estudiantes dominicanos de primer año universitario que realizaron la prueba de léxico disponible sobre los 16 centros tradicionales. El objetivo del autor era conocer el "mayor o menor grado de coincidencia en las respuestas" (Alba 1995: 19). Para calcularlo, es necesario dividir en cada centro el total de palabras por el número de vocablos.

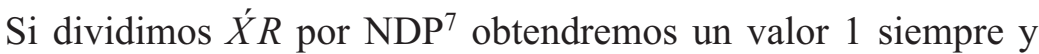
cuando todos los sujetos hayan respondido las mismas palabras en su encuesta léxica, cosa por lo demás quimérica. Por el contrario, cuanto más variadas sean las respuestas, menor será el índice de cohesión; es decir, la dispersión es mayor. De este modo nuestro índice de cohesión informa sobre si nuestro centro de interés es cerrado o compacto, o más bien abierto o difuso (Echeverría 1991: 62).

Bartol (2004) y Hernández Muñoz (2006) dejan patente que el número de informantes condiciona el número de vocablos $\mathrm{y}$, por ende, afecta directamente al cálculo de ambos índices ya que "se debe tener en cuenta que el índice de cohesión está condicionado por el número de vocablos y, por consiguiente, por las normas de edición" (Bartol 2004: 51). De hecho, como apuntan Fernández Juncal y Hernández Muñoz (2019: 185), "las categorías semánticas pueden ser de diferente naturaleza, así como el número de ejemplares que las constituyen y los límites que las definen, desde las categorías clásicas aristotélicas a las categorías de límites difusos de los modelos cognitivos". En este sentido, los diferentes resultados obtenidos (número de palabras, número de vocablos e índice de cohesión) permiten situar en una

7. Donde $X$ R hace referencia el promedio de respuestas ofrecidas por un informante y NDP al número de palabras diferentes. 
escala los centros mostrándolos en una clasificación por rangos. Estas premisas hacen que, paulatinamente, hayan ido surgiendo estudios que incorporan otros criterios de clasificación (Hernández Muñoz 2006; López González 2014; Sánchez-Saus 2011; 2013; 2016; 2019; Tomé 2011 ; 2015) o que aluden a las taxonomías previamente establecidas (Del Barrio 2018; Gómez Devís 2019) para analizar el lexicón mental de sus informantes.

En primer lugar, Hernández Muñoz (2006) distingue dos tipos de centros según los niveles de inclusividad: centros inclusivos y centros relacionales. Los primeros están formados por elementos que mantienen una asociación directa con la categoría y donde aplica la ecuación " $\mathrm{x}$ es Y". Por ejemplo, "la mano es una parte del cuerpo". En cambio, en los centros relacionales los elementos no se relacionan de forma tan evidente con la categoría. En este caso, enuncia la proposición "x es parte de $Y$ " $y$, más precisamente, "x está relacionado con $Y$ " o "x tiene algún tipo de relación con Y". Por ejemplo, "las Matemáticas están relacionadas con la escuela” (Hernández Muñoz 2006: 151-152).

Este estudio, junto con los de Tomé (2011; 2015), permitió establecer cinco tipos de categorización de los centros de interés tomando como referencia la estructura interna de estos en su dimensión horizontal: natural, bien definida, ad hoc, radial (Hernández Muñoz 2006) y esquema (Tomé 2011; 2015):

a) Por categoría natural entiende aquellas que presentan las características clásicas de una categoría semántica justificada deontológicamente por la teoría de los prototipos, la teoría de los ejemplares o la teoría del conocimiento (Hernández Muñoz 2006: 135). Se enmarcan en este grupo los centros: $L a$ ropa; Muebles de la casa; Alimentos y bebidas; La cocina y sus utensilios; Calefacción, iluminación y medios para airear un recinto; Medios de transporte; Trabajos del campo y del jardin; Los animales; Profesiones y oficios.

b) La categoría bien definida se corresponde con los centros que tienen una estructura clara y cerrada, como es Partes del cuerpo humano. 
c) La categoría ad hoc-o derivada de metas-está compuesta por elementos que se organizan en torno a una finalidad o propósito, como, por ejemplo: enumerar los cubiertos necesarios para comer. Así, se incluye dentro de este grupo el centro de interés: Objetos colocados en la mesa para la comida.

d) La categoría radial se configura en forma de red en la que existen subcategorías compuestas por diferentes elementos periféricos, como es el caso del centro de interés Juegos y distracciones.

e) Por último, la categoría esquema está basada en la distinción de Richards (1969). Los elementos están relacionados con los espacios del mundo real que el informante puede describir desde diferentes ópticas: objetos, sentimientos, personas, etc. Los centros organizados según esta categoría serían: La cocina y sus utensilios; La escuela (muebles y materiales); La ciudad; El campo; y Juegos y distracciones.

Más adelante, tomando como referencia las ideas de Hernández Muñoz (2006) y Tomé Cornejo (2011), Sánchez-Saus (2011) realiza una nueva taxonomía simplificada distinguiendo tres grupos: categorías naturales, categorías radiales y categorías esquema. Dentro de este último grupo define una subcategoría: el guion, y lo atribuye al centro denominado Acciones y actividades habituales por estar más relacionado con lo eventual y con la secuenciación de acciones.

Por su parte, López González (2014b) recoge las aportaciones de Hernández Muñoz (2006), Tomé (2011) y Sánchez-Saus (2011) y observa que la clasificación de la categoría radial y el esquema coinciden en todos los centros de interés, por lo que, desde su punto de vista, no tendría sentido tal distinción en los centros de interés tradicionales. Asimismo, asocia el centro de interés El cuerpo humano tanto a la categoría natural como a la bien definida.

Como se aprecia, las características internas de los centros de interés han sido ampliamente abordadas en lengua española. Sin embargo, no se consignan estudios dedicados específicamente a este fin en lengua extranjera: en inglés, Ferreira y Echeverría (2010) se aproximan a las redes semánticas y Ferreira, Garrido y Guerra (2019) 
se centran en las variables más incidentes en la configuración del lexicón mental de 60 estudiantes chilenos de inglés avanzado; Lugones (2015) se interesa por el léxico de estudiantes bilingües de Educación secundaria; en francés, De la Maya (2015) se ocupa de la adquisición de la competencia léxica de una segunda lengua. Así, el presente trabajo dará inicio a esta línea de investigación que posibilitará la realización de comparaciones ulteriores con una metodología común.

\section{Objetivos y planteamiento inicial}

El objetivo principal de este trabajo es proponer una taxonomía a partir de la creación de un índice estructural de los centros de interés considerados en el presente estudio. De este objetivo principal se desprenden los siguientes objetivos específicos: O1. Demostrar el diferente comportamiento de los centros de interés tanto en español -lengua materna- como en inglés y francés -lengua extranjera- y O2. Establecer una medida de valoración de sus características.

De esta forma, la propuesta de clasificación de los centros de interés que se propone en el presente estudio se basa en la organización de las palabras en la mente distinguiendo entre centros prototípicos y centros relacionales. Los primeros hacen referencia a centros homogéneos cuyo léxico nuclear se encuentra mejor definido. En cambio, los segundos son menos compactos y las relaciones entre las palabras están menos organizadas. No se trata de una dicotomía entre centros prototípicos y centros relacionales sino más bien de localizar los dos extremos de un continuo en el que se sitúan los centros de interés.

A grandes rasgos, los centros prototípicos suelen coincidir con los centros naturales y los centros relacionales con los centros radiales pero la distinción se ha realizado adecuando la teoría a los resultados obtenidos en este estudio. No obstante, existen ciertas divergencias ya que se ha pretendido el esbozo de una taxonomía que refleje la estructura interna de los centros atendiendo a tres criterios: la centralidad, la cohesión y la conectividad de los vocablos. Si bien la clasificación está influida por el número de informantes y sus características, se parte de la hipótesis de que el estímulo es el principal responsable de la estructura interna de cada centro de interés. 


\section{Método}

\section{Participantes}

La población de estudio se compone de 171 estudiantes matriculados en el Máster en Profesorado de Educación Secundaria Obligatoria y Bachillerato, Formación Profesional y Enseñanza de Idiomas de la Universidad de Málaga en la modalidad de evaluación ordinaria -con asistencia obligatoria-. 117 son mujeres y 54 hombres. Los estudiantes tuvieron que acreditar en la fase de preinscripción un dominio de una lengua extranjera equivalente al nivel B1 del Marco Común Europeo de Referencia.

Inicialmente existe un 8,5\% de error de muestreo que corresponde a la relación entre los 171 elementos de la muestra y los 385 de la población total. Sin embargo, aunque totalidad de la población $(\mathrm{N}=$ 385), considerada como los estudiantes matriculados, no coincide con la muestra $(\mathrm{n}=171)$, se dota a la muestra un carácter censal pues está constituida por informantes representativos y válidos. De esta forma, los errores aleatorios de muestreo desaparecen, siendo los errores sistemáticos aquellos que únicamente podrían alterar las estimaciones presentadas. Por ello, Siguiendo la propuesta de Dörnyei y Csizér (2012: 82), los cuestionarios se pasaron a todo el alumnado del Máster accesible, estratificado en relación con las especialidades: -Vid. Tabla 1-.

Tabla 1 - Distribución de los informantes que conforman la muestra de estudio por especialidad

\begin{tabular}{|l|c|}
\hline Especialidad & Participantes \\
\hline Dibujo, Imagen y Artes Plásticas & 11 \\
\hline Formación y Orientación Laboral & 7 \\
\hline Ciencias Sociales, Geografía e Historia & 17 \\
\hline Hostelería y Turismo & 10 \\
\hline Lengua extranjera & 38 \\
\hline Lengua, Literatura, Latín y Griego & 19 \\
\hline Música & 9 \\
\hline Orientación Educativa & 10 \\
\hline Tecnología, Informática y Procesos Industriales & 40 \\
\hline Matemáticas & 10 \\
\hline Total & 171 \\
\hline
\end{tabular}




\section{Instrumentos}

Para la recogida de los datos se utilizaron dos instrumentos diferenciados pero complementarios: una prueba de léxico disponible, que permitió evaluar la competencia léxica de los informantes; y un cuestionario sociodemográfico, que se inicia con un apartado para recoger datos identificativos para conocer el grupo, el idioma en el que se realiza la prueba y la fecha exacta en que se realizó. A continuación, aparecen 27 ítems sociológicos para compilar la información relacionada con la formación y con el contacto con otras lenguas tanto del informante como de sus familiares. Además, se incluyen preguntas relacionadas con el nivel económico del informante, su movilidad geográfica y con la densidad de sus relaciones.

La metodología seguida para la recopilación del léxico se ha ajustado a las directrices marcadas por el PPHDL (López González 2014a): a continuación del cuestionario sociológico, aparecían 10 folios en blanco grapados. Los investigadores iban nombrando cada centro de interés y los informantes disponían de dos minutos para evocar, por escrito, todas las palabras que venían a su mente, sin prestar atención a los aspectos ortográficos.

Concretamente, se han seleccionado nueve centros de interés en español -al ser la lengua materna de los informantes- y en lengua extranjera-inglés ( $\mathrm{n}=150)$ o francés $(\mathrm{n}=21)$, pues todos los estudiantes afirmaron haber acreditado un nivel mínimo de B1 en alguno de estos idiomas para acceder al máster-.

Los centros de interés seleccionados corresponden a 8 tradicionales presentes en el estudio de Gougenheim et al. (1956), como son: Partes del cuerpo humano; La ropa; Comidas y bebidas; La escuela (muebles y materiales); La ciudad; Medios de transporte; Juegos y distracciones; Profesiones y oficios. Además, se incorporó un noveno centro de interés relacionado con una de las competencias presentes en la profesión docente como Ordenadores e internet.

\section{Procedimiento}

Una vez recopilado el léxico de los informantes en lengua materna y lengua extranjera, se procedió a la edición de los materiales siguiendo 
las directrices marcadas por Samper Padilla et al. (2003) en una matriz diseñada ad hoc. Igual que ocurre en las investigaciones llevadas a cabo por Sánchez-Saus (2016) y Tomé (2015), los criterios de edición han permitido la inclusión de todo tipo de palabras que el informante ha relacionado con el centro de interés difuminando los límites de las categorías naturales y asemejándolas a las categorías radiales. Por esta razón, aunque Partes del cuerpo humano sea una categoría bien definida y un centro de interés en el que los investigadores encuentran escasas dificultades para decidir qué palabras incluir (Ayora 2006; Carcedo 2000; Galloso 2003; Gómez Devís 2003; Hernández Muñoz 2004; Samper Padilla 1998; Tomé 2015; Trigo 2011, entre otros), esa propiedad no se refleja en los resultados ya que se han incluido vocablos en ese centro de interés como hidrato o dieta que no son en sí mismos partes del cuerpo, sino que son fruto de asociaciones indirectas. Sin embargo, sin unos criterios de edición abiertos, no se lograría que los resultados fueran el fiel reflejo del lexicón mental de los informantes en cada uno de los centros de interés abordados.

Para obtener los valores que serán utilizados en la clasificación propuesta en este estudio, se utilizaron diferentes programas. En primer lugar, la matriz se procesó en el programa Lexidisp (Moreno, Moreno y García 1995) y se obtuvieron índices generales como el número de palabras y vocablos por centro de interés presentes en el estudio de Santos Díaz (2020). Posteriormente, se otorgó un valor a cada palabra de la matriz según su relación con el centro de interés siguiendo la propuesta de Ávila y Villena (2010): 1 para las palabras nucleares, 0,5 para las asociaciones colectivas, 0,25 para las derivaciones y 0 para las asociaciones individuales. Esos datos se exportaron al programa estadístico IBM SPSS Statistics 22 y se realizaron análisis descriptivos para conocer la frecuencia de las palabras nucleares por centro de interés. Finalmente, con el programa DispoGrafo (Echeverría, Vargas, Urzúa y Ferreira 2008) se obtuvieron el número de conexiones en cada centro de interés y el peso o fuerza de cada una de ellas.

Para la clasificación según rangos de los centros en función de su estructura interna se ha utilizado el índice estructural definido como la suma del índice de cohesión, el índice de centralidad y el índice de conectividad. El índice de cohesión muestra la relación entre la media de palabras por informante y el número total de vocablos. Este 
índice oscila de 0 a 1 de forma que cuanto más se acerque a 1 más homogéneo será el vocabulario y viceversa, cuanto más se acerquen a 0 más heterogéneas serán las respuestas de los informantes.

Tomando como referencia el número de vocablos nucleares, se ha generado el índice de centralidad que muestra la cantidad de vocablos nucleares en cada centro con respecto al total de vocablos. Este indicador se obtiene al dividir el número total de vocablos por el número de vocablos considerados centrales o nucleares. El índice oscila entre un máximo de 1 , correspondiente a un número total de palabras y vocablos nucleares idéntico, y un mínimo de 0 , en el supuesto de que no existiese ningún vocablo nuclear.

$$
\text { Índice de centralidad }=\frac{\sum V}{\sum V N}
$$

En el índice de conectividad se ha tenido en cuenta el número de conexiones con independencia del peso o la frecuencia de estas. Obviamente, si se consideran todas las conexiones y se suman los pesos, el resultado expresará que el total de palabras está relacionado, ya que se considera la relación unidireccional de una sola palabra por vocablo y eso siempre se produce. Por ello, se ha considerado el número de conexiones entendiendo que cuanto menor sea el número de conexiones con respecto al número de palabras aportadas, mayor será la fuerza de la conexión entre esas palabras. El índice de conexión se obtiene al dividir el número total de conexiones por el número de palabras y restarle 1.

En la primera operación se obtiene el número de relaciones que se han producido por cada palabra que el informante ha aportado. Dentro de las relaciones entre palabras se han sumado todas aquellas diferentes, por lo que cuanto menor sea el número de relaciones mayor será su peso o fuerza. El índice oscila entre 0 y 1 , donde 0 equivaldría a ninguna relación por palabra (lo cual es imposible) y 1 a que todas las palabras mantienen relaciones diferentes. Para poder realizar una comparativa con el índice de centralidad y de cohesión en los que se muestra la homogeneidad del núcleo, se ha restado 1 al resultado de forma que los datos que se acercan a 1 muestran conexiones con mayor 
peso en las palabras y los resultados que se acercan a 0 relaciones más difusas.

$$
\text { Índice de conexión }=1-\left(\frac{\sum C}{\sum P}\right)
$$

\section{Resultados}

La Tabla 2 muestra los resultados ordenados según el rango que ocupan los centros de interés en cada índice. En cada idioma, se han destacado en sombreado los tres primeros centros y los tres últimos, de forma que se establecen tres intervalos según su homogeneidad.

Tabla 2 - Indicadores de la estructura de los centros de interés según idioma ${ }^{8}$

\begin{tabular}{|ccccccccc|}
\hline Lengua & CI & $\begin{array}{c}\text { Índice de } \\
\text { cohesión }\end{array}$ & CI & $\begin{array}{c}\text { Indice de } \\
\text { centra- } \\
\text { lidad }\end{array}$ & CI & $\begin{array}{c}\text { Índice de } \\
\text { conecti- } \\
\text { vidad }\end{array}$ & CI & $\begin{array}{c}\text { Índice } \\
\text { estructural }\end{array}$ \\
& 1 & 0,108 & 1 & 0,176 & 6 & 0,554 & 1 & 0,832 \\
& 2 & 0,092 & 2 & 0,172 & 1 & 0,548 & 2 & 0,791 \\
& 6 & 0,081 & 6 & 0,147 & 2 & 0,526 & 6 & 0,782 \\
& 4 & 0,055 & 8 & 0,146 & 4 & 0,471 & 4 & 0,655 \\
& 3 & 0,05 & 5 & 0,139 & 3 & 0,330 & 3 & 0,518 \\
& 5 & 0,045 & 3 & 0,138 & 5 & $0,319 *$ & 5 & 0,503 \\
& 8 & 0,044 & 7 & 0,136 & 7 & 0,319 & 7 & 0,493 \\
& 7 & 0,038 & 4 & 0,128 & 9 & 0,314 & 8 & 0,475 \\
& 9 & 0,035 & 9 & 0,124 & 8 & 0,284 & 9 & 0,473 \\
\hline
\end{tabular}

8. Los centros de interés en español son: 1. Partes del cuerpo humano, 2. La ropa, 3. Comidas y bebidas, 4. La escuela (muebles y materiales), 5. La ciudad, 6. Medios de transporte, 7. Juegos y distracciones, 8. Profesiones y oficios y 9. Ordenadores e internet; en inglés: 1. Human body parts, 2. Clothes, 3. Food and drink, 4. The school (furniture and school material), 5. The city, 6. Means of transport, 7. Games and entertainments, 8. Professions and jobs, 9.Computers and Internet; en francés: 1. Parties du corps humain, 2. Les vêtements, 3. La nourriture et les boissons, 4. L'école (meuble et matériel), 5. La ville, 6. Moyens de transport, 7. Jeux et loisirs, 8. Professions et métiers, 9. Ordinateurs et Internet.

* Con objeto de que los valores mayores indiquen más homogeneidad y prototipicidad se ha restado uno de forma que los centros con un índice más elevado sean los que dispongan de relaciones más fuertes. 


\begin{tabular}{|ccccccccc|} 
Lengua & CI & $\begin{array}{c}\text { Índice de } \\
\text { cohesión }\end{array}$ & CI & $\begin{array}{c}\text { Índice de } \\
\text { centra- } \\
\text { lidad }\end{array}$ & CI & $\begin{array}{c}\text { Índice de } \\
\text { conecti- } \\
\text { vidad }\end{array}$ & CI & $\begin{array}{c}\text { Índice } \\
\text { estructural }\end{array}$ \\
\hline \multirow{6}{*}{ Inglés } & 1 & 0,149 & 1 & 0,226 & 1 & 0,586 & 1 & 0,961 \\
& 2 & 0,092 & 2 & 0,185 & 2 & 0,508 & 2 & 0,785 \\
& 6 & 0,068 & 6 & 0,174 & 6 & 0,372 & 6 & 0,761 \\
& 3 & 0,067 & 4 & 0,131 & 4 & 0,469 & 4 & 0,658 \\
& 4 & 0,058 & 3 & 0,157 & 3 & 0,303 & 3 & 0,614 \\
& 8 & 0,046 & 8 & 0,158 & 8 & 0,536 & 8 & 0,518 \\
& 5 & 0,046 & 7 & 0,145 & 7 & 0,322 & 7 & 0,508 \\
& 7 & 0,041 & 5 & 0,157 & 5 & 0,315 & 5 & 0,506 \\
& 9 & 0,033 & 9 & 0,117 & 9 & 0,280 & 9 & 0,430 \\
& 1 & 0,261 & 6 & 0,302 & 6 & 0,353 & 6 & 0,879 \\
& 6 & 0,224 & 1 & 0,282 & 1 & 0,265 & 1 & 0,807 \\
& 2 & 0,217 & 2 & 0,275 & 2 & 0,255 & 2 & 0,747 \\
& 5 & 0,137 & 5 & 0,237 & 4 & 0,201 & 4 & 0,559 \\
& 4 & 0,136 & 8 & 0,235 & 7 & 0,152 & 5 & 0,495 \\
& 3 & 0,130 & 3 & 0,223 & 9 & 0,152 & 3 & 0,494 \\
& 8 & 0,120 & 4 & 0,221 & 3 & 0,142 & 9 & 0,491 \\
& 9 & 0,118 & 9 & 0,220 & 8 & 0,127 & 8 & 0,482 \\
& 7 & 0,107 & 7 & 0,2 & 5 & 0,121 & 7 & 0,460 \\
\hline
\end{tabular}

Se constata la similitud entre las respuestas de un mismo centro en los tres índices. Así, en español 1) Partes del cuerpo humano ocupa el primer lugar en cuanto al índice de cohesión, índice de centralidad y en el índice estructural, pero ocupa la segunda posición en el índice de conectividad (con un índice de 0,548 muy similar al centro con mayor conectividad, 6) Medios de transporte, que cuenta con un índice de $0,554)$.

Los centros de interés Partes del cuerpo humano; La ropa; y Medios de transporte, se sitúan en las tres primeras posiciones del rango. Según la clasificación de Hernández Muñoz (2006), el primer centro es considerado una categoría bien definida y los otros dos, categorías naturales según su estructura interna, pero los tres son inclusivos según su nivel de inclusividad. Medios de transporte ocupa el tercer puesto en cuanto a índice de cohesión e índice de conectividad. Se trata del centro con el menor número de vocablos, lo que indica que el núcleo está mejor definido. En cambio, las relaciones entre las palabras 
son más diversas que en los dos centros primeros, por lo que ocupa el quinto lugar. Estos resultados coinciden con los de Tomé (2015).

En el nivel intermedio se sitúan los centros La escuela: muebles y materiales, Comidas y bebidas y La ciudad. La escuela es un centro clasificado en Hernández Muñoz (2006) como una categoría radial y como categoría esquema por Tomé (2015). De hecho, en el índice de nuclearidad ocupa el penúltimo lugar. En cambio, en el índice de cohesión y el índice de conectividad ocupa el cuarto lugar. Ello indica que, aunque el núcleo sea algo más amplio, las palabras están cohesionadas y conectadas.

Comidas y bebidas, clasificado como natural por Hernández Muñoz (2006) y Tomé (2015), presenta un núcleo con una proporción de palabras superior por lo que ocupa el sexto lugar. No obstante, en el índice de cohesión se sitúa en el quinto lugar y en el índice de conectividad en el tercero. Se trata de un centro de interés con una estructura bien definida y con unas relaciones claras, aunque también sea un centro donde, en ocasiones, los informantes aportan nombres específicos de comidas o realizan asociaciones individuales que llevan a desviarse del núcleo.

La ciudad es un centro clasificado como radial y de categoría relacional por Hernández Muñoz (2006) y como esquema por Tomé (2015). Ocupa el quinto lugar en el índice de nuclearidad, pero se sitúa en el sexto en cuanto al índice de cohesión y el séptimo en el índice de conectividad. Se trata de un centro muy productivo y con una gran riqueza léxica ya que es un campo abierto en el que los límites del prototipo son bastante difusos.

En el extremo inferior se sitúan los centros: juegos y distracciones; Profesiones y Oficios y Ordenadores e internet. El primero es un centro claramente relacional (radial en Hernández Muñoz 2006 y Tomé 2015), en el que los límites no están tan bien definidos debido a que existen muchas formas de pasar el tiempo libre y distraerse que dependen directamente de los informantes y de sus características (por ejemplo, el golf aparecerá con más frecuencia en los informantes con un poder adquisitivo mayor). En el índice de centralidad ocupa el séptimo lugar, en el índice de cohesión el octavo y en el de conectividad de los vocablos el sexto lugar. La variedad de respuestas en este centro hace 
que las relaciones entre las palabras sean más diversas y el prototipo esté más disperso.

Profesiones y oficios, incluido dentro de la categoría natural por Hernández Muñoz (2006) y Tomé (2015), es un centro en el que se ha producido una serie de asociaciones indirectas que llevan a aportar más vocablos diferentes, lo que sitúa en el séptimo puesto del rango según el índice de cohesión. En cambio, la proporción de vocablos nucleares con respecto al total de vocablos es mayor y se sitúa en el cuarto puesto, lo que indica que es un centro cuyo núcleo está bien definido. El hecho de que los estudiantes sean de postgrado y se hayan incorporado o lo vayan a hacer en breve, quizás ha llevado a un mayor conocimiento de distintas profesiones y a asociaciones indirectas e incluso individuales.

El centro 9) Ordenadores e internet -no considerado en estudios precedentes- se sitúa en el índice de cohesión, de centralidad y estructural en el último rango. Tan solo sube un puesto en el índice de conectividad gracias a relaciones entre palabras muy frecuentes (por ejemplo, teclado y ratón han sido aportadas en uno u otro orden por 75 informantes). El ordenador podría considerarse un centro inclusivo con una estructura natural si el informante se limitara a enumerar sus partes, ya que si se abre el campo a nombres de programas informáticos o software la lista sería encomiable. Asimismo, los límites de internet son muy amplios lo que lleva a que se trate de un centro relacional donde se incluyan relaciones de todo tipo (nombres propios de páginas webs, nombres de programas, etc.).

En inglés, la distribución de los centros según rango es semejante, pero está mejor definida. Se observa que hay cinco centros que repiten posición en los cuatro índices: 1) Human body parts; 2) Clothes; 6) Means of transport; 8) Jobs and professions y 9) Computer and Internet. En los tres primeros lugares se sitúan los mismos centros que en español (Human body parts, Clothes, Means of transport). A continuación, se incluyen los centros intermedios en los que se ha cambiado el centro 5) The city; por el 8) Jobs and professions. Esto puede deberse a que en el aprendizaje de una lengua extranjera existe más vocabulario relacionado con la ciudad que con las profesiones, razón por la cual los informantes han aportado más variedad de unidades léxicas. 
En francés, los tres primeros centros coinciden con la clasificación en español y en inglés: 1) Parties du corps humain, 6) Moyens de transport y 2) Les vêtements). Los tres que se sitúan en la parte central de la escala son los mismos que en español. No obstante, se han sombreado los centros que se sitúan en su gran parte en el subgrupo formado por los centros con índices menores. Cabe destacar que el centro 9) Ordinateurs et Internet, al igual que en español, no se sitúa en el último rango según conectividad quizás debido a la existencia de conexiones muy frecuentes y que este centro no cuenta con la misma riqueza léxica que en inglés.

En cambio, el quinto centro -La ville-presenta una estructura más homogénea en francés que en inglés, aunque ocupa el último rango según conectividad. Como se comentó anteriormente, los índices están influenciados por el número de informantes y en este caso el número de informantes es considerablemente menor, por lo que quizás los datos no se encuentren tan bien definidos como en español o inglés. Por ello, la comparación se hace en función de los rangos que ocupan los centros en cada índice con independencia del número exacto en cada caso.

El objetivo de este apartado ha sido demostrar el diferente comportamiento de los centros de interés y establecer una medida de valoración de sus características. De la comparación de las similitudes y divergencias en la clasificación en cada idioma se pueden extrapolar algunos resultados generales compartidos en gran medida en los tres estudios. A modo de ejemplo, se presenta el continuo que formarían los centros ordenados según el rango español. En el extremo izquierdo se sitúa el centro más prototípico, 1) Partes del cuerpo humano, y en el derecho el centro menos prototípico o relacional, es decir, con una estructura más abierta a relaciones y asociaciones diferentes, 9) Ordenadores e internet. 


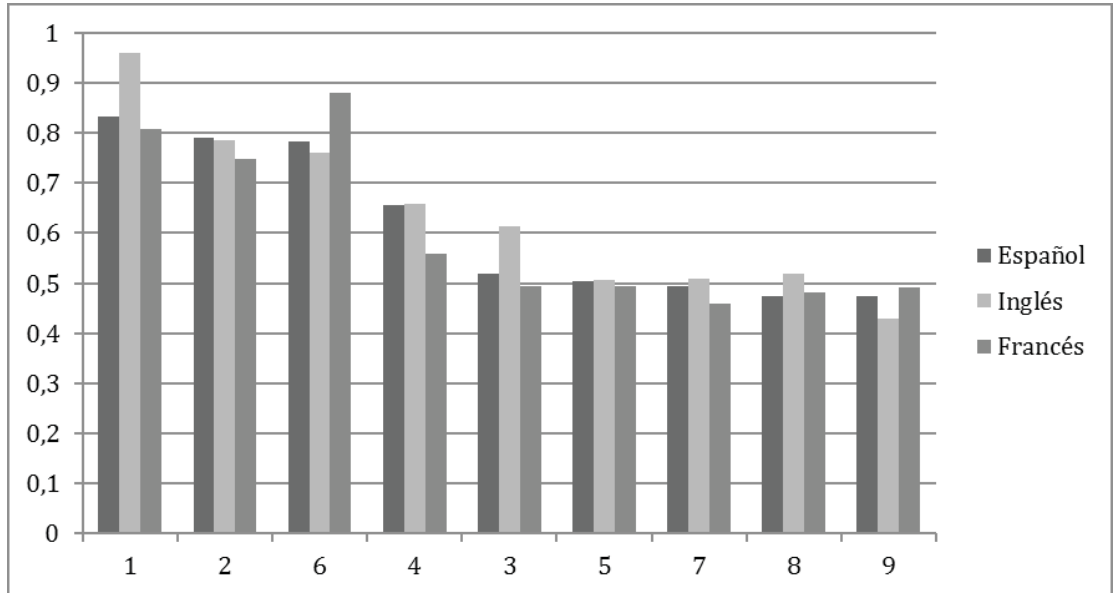

Gráfico 1 - Escala de prototipicidad de los centros de interés

En el Gráfico 1 se destacan los tres primeros centros que podrían clasificarse como prototípicos: 1) Partes del cuerpo humano, 2) La ropa y 6) Los medios de transporte. A continuación, le siguen los centros que podrían clasificarse como relacionales, empezando por el 4) La escuela: muebles y materiales. Los cinco últimos centros de interés presentan resultados similares en los tres idiomas a excepción del centro de 3) Comidas y bebidas que tiene en inglés un índice ligeramente superior a los otros dos idiomas, pero por debajo del centro que le precede en la clasificación.

\section{Propuesta una taxonomía a partir de la creación de un índice estructural}

A la luz de los resultados generales en los tres idiomas, queda patente la necesidad de diferenciar los resultados según los centros de interés debido a su diferente ontogenia. Quizás si se comparara la media de palabras por informante y centro de interés de los nueve centros de este estudio con otra investigación que incluyese los dieciséis tradicionales, la media de los primeros sería superior debido a que se han eliminado centros de interés poco productivos como Trabajos del campo y del jardín o Iluminación, calefacción y medios de airear 
un recinto. Hasta ahora se ha comparado el promedio de respuestas entre estudios con independencia de los centros de interés utilizados ya que según Bartol (2001: 223) es el único índice que neutraliza las diferencias en el número de informantes.

No obstante, lo recomendable sería solamente comparar el promedio de respuestas de los estudios cuyo estímulo sea el mismo. Por ejemplo, Hernández Muñoz (2006: 314) contrasta la media en cada centro de interés de 17 muestras diferentes según los denominados centros tradicionales. En cambio, la autora afirma que no es igual la denominación La escuela que La escuela: muebles y materiales. En el caso de la muestra de estudiantes de Castilla la Mancha el estímulo ha sido La escuela dejando el campo léxico más abierto a posibles relaciones. En cambio, los estudios que han incluido muebles y materiales cierran más el campo de actuación de los informantes provocando que algunos escriban menos palabras. De hecho, Hernández Muñoz modifica la clasificación según la inclusividad del centro: si se trata de La escuela es un centro relacional y si se refiere a La escuela: muebles y materiales es un centro inclusivo.

En los resultados comparativos de cada idioma se ha mostrado que las respuestas son similares según el campo en cuestión. Esto supondría que la media de palabras calculadas por centro e informante en cada lengua no fuera comparable con estudios que incluyan otros centros de interés, ya que, coincidiendo con lo expuesto por Bartol (2001) y Hernández Muñoz (2006), apreciamos que del estímulo depende en gran medida el producto $\mathrm{y}$, por ende, el número de palabras y vocablos.

La clasificación propuesta en este estudio no pretende diferenciar de forma contundente la estructura de un centro con respecto a otro, sino más bien elaborar una escala en la que cada centro pueda situarse según su prototipicidad. Para ello, toma como referencia los fundamentos teóricos presentes en los estudios de Hernández Muñoz (2006), Tomé (2011), Sánchez-Saus (2011), López González (2014b) y como herramienta de análisis los resultados de las pruebas de disponibilidad léxica.

Concretamente, los principios en los que se basa la taxonomía realizada son: la cohesión del centro, la prototipicidad del núcleo y las 
relaciones entre las palabras. Cada característica da lugar a un índice que lo cuantifica: el índice de cohesión muestra la relación entre la media de palabras por informante y el número de vocablos; el índice de centralidad cuantifica la cantidad de vocablos nucleares con respecto al total de vocablos; el índice de conectividad mide la fuerza de las relaciones entre los vocablos al dividir el número de conexiones por el número total de palabras. El primer índice acredita que cuanto mayor sea el índice de cohesión más homogénea será la muestra; el segundo indica que el núcleo estará más o menos definido; el tercero determina la fuerza de las conexiones entre las palabras. El compendio de esos tres índices da lugar al índice estructural que, como su nombre indica, sirve para definir la estructura de los centros de interés.

Índice estructural $=I C+I C e+I C o$

donde

IC es el índice de cohesión

$I C e$ es el índice de centralidad

ICo es el índice de conectividad

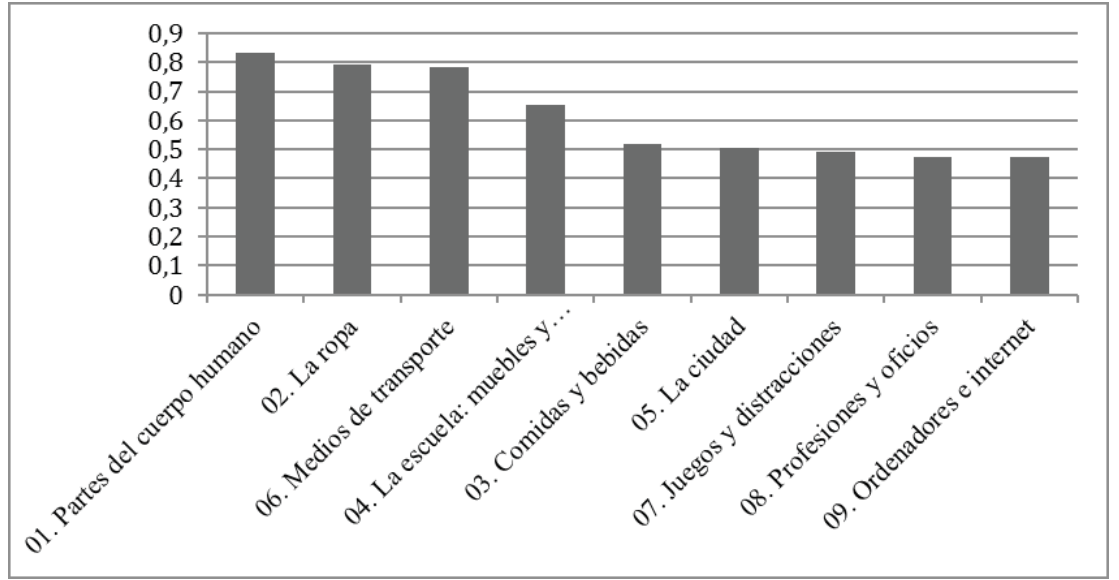

Gráfico 2 - Clasificación de los centros de interés de este estudio según el índice estructural

Si se realizara una personificación de los centros, se podría decir que son como familias: hay familias en las que el prototipo es más o 
menos numeroso y otras en las que se invita a conocidos que guardan cierta relación directa o indirecta. Cuanto menor sean los miembros de la familia, mayor comunicación y relación habrá entre ellos, como es el caso de 1) Partes del cuerpo humano. En cambio, en 6) Medios de transporte los vocablos o miembros de la familia son menos numerosos, pero no han acudido con tanta frecuencia y cuentan con un menor número de palabras o producciones léxicas, razón por la cual se considera un centro de interés menos prototípico.

Al igual que ocurre en las familias, resulta complicado discernir cuál es el modelo más común. Por ello, se ha acudido a la estadística para que, igual que es posible conocer la media de hijos por familia, sea posible cuantificar en cada centro el léxico nuclear y diferenciarlo del resto. Para completar esta clasificación, se han analizado las principales relaciones del lexicón mental según las características de los centros de interés basadas en las dos teorías que, según Hernández Muñoz (2006), están presentes en los estudios de disponibilidad léxica: la teoría de los prototipos y la teoría de las redes semánticas.

\section{Discusión}

La aplicación de los estudios de disponibilidad léxica a la didáctica de la lengua ha quedado ampliamente recogida en diversas investigaciones (Paredes 2012; Tomé 2015; Hidalgo 2017; SánchezSaus 2016; Santos Díaz 2017; Trigo, Romero y Santos Díaz, 2018, 2019, 2020; Santos Díaz, Trigo y Romero, 2020; entre otras). En esta línea, el conocimiento de la naturaleza interna de los distintos centros de interés es un factor crucial para realizar una adecuada selección léxica de cara a la planificación de la enseñanza de idiomas, como así lo viene apuntando un número creciente de investigadores (Paredes 2015; Ávila 2016; Santos Díaz 2017; Hidalgo 2019). En consonancia con esta idea, se ha comprobado que, desde los inicios de la disponibilidad léxica, los autores franceses Gougenheim et al. (1964: 189-190) destacaron el diferente comportamiento de los centros de interés según el número de palabras y vocablos aportados por los informantes. A priori, distinguieron entre niveles de homogeneidad y pusieron de manifiesto la mayor idoneidad de unos centros con respecto a otros debido a los niveles de disponibilidad que presentaban. 
Esa distinción está presente en la mayoría de los resultados comparativos generales de las investigaciones donde se organizan los centros según el número de palabras y vocablos aportados y el índice de cohesión (Carcedo 2000; Samper Hernández 2002; Ávila y Villena 2010; Bellón 2011, Trigo 2011, entre otros). Algunos estudios, como los de Hernández Muñoz (2006), Tomé (2011; 2015) y Sánchez-Saus (2019), presentan una clasificación atendiendo a la estructura interna de los centros. Otros estudios, aunque no llegan a ofrecer una taxonomía, hacen alusión a la estructura interna de los centros y, en función de ella, proponen la inclusión o exclusión de determinados centros de interés en función del tipo de estudio abordado (González Fernández 2014; Paredes 2014; Hidalgo 2017).

Tras analizar las taxonomías de los estudios anteriores y los diferentes resultados generales, se ha propuesto una clasificación de los centros de interés según su prototipicidad. El objetivo es conocer el comportamiento de cada uno a través del compendio de tres índices: el índice de cohesión, que mide la relación del promedio de palabras de un informante en un centro entre los vocablos de ese centro; el índice de centralidad que muestra la distribución de los vocablos nucleares con respecto a los vocablos totales de un centro; y el índice de conectividad que da cuenta de la mayor o menor solidez de las relaciones de los vocablos de un centro. Este último se obtiene restando uno a la cantidad resultante de dividir el número de conexiones diferentes entre el total de palabras.

El resultado es un índice estructural que da cuenta de la homogeneidad del centro, de la organización de las palabras nucleares y de la fuerza de las conexiones entre los vocablos. Esta expresión numérica servirá para organizar los centros en una escala cuyos extremos están formados por el centro más prototípico, 1) Partes del cuerpo humano, y el centro más relacional o lo que es lo mismo, menos prototípico, 9) Ordenadores e internet. El resto de los centros se posiciona en ese continuo que ayuda a definir su estructura. Así, 2) La ropa y 6) Medios de transporte se acercan al grupo de centros prototípicos, 4) La Escuela: muebles y materiales se ubica en un nivel intermedio y el resto de los centros -3) Comidas y bebidas, 5) La ciudad, 7) Juegos y distracciones y 8) Profesiones y oficios-muestran resultados semejantes al centro de interés más relacional. 
Esa clasificación es muy similar en los tres idiomas del estudio e incluso con un número desigual de informantes en cada uno de ellos ( $\mathrm{N}=171$ en español; $\mathrm{n}=150$ en inglés y $\mathrm{n}=21$ en francés). Por tanto, aunque esta propuesta podría verse alterada por factores psicolingüísticos -como el nivel de instrucción o el conocimiento de idiomas-y sociolingüísticos -como el sexo, la ubicación geográfica o la edad- también permite atisbar que será muy parecida en otros estudios. Este esbozo de la taxonomía de los centros viene a completar las clasificaciones teóricas con resultados prácticos, ya que este estudio no se ha limitado a incluir los vocablos que mantienen una relación directa con el centro de interés, sino que se han incorporado todas las producciones de los individuos con independencia del grado de relación con el prototipo.

Retomando las conclusiones de los investigadores franceses (Gougenheim et al. 1964), resulta curioso que, investigaciones con casi medio siglo de diferencia, kilómetros de distancia y lenguas diferentes, coincidan en que los centros de interés más homogéneos en su caso o prototípicos en este estudio sean 1) Partes del cuerpo humano y 2) La ropa. Gracias a la elaboración de un índice estructural, es posible conocer qué tipo de configuración presenta cada centro de interés y los posibles modelos de asociación léxica que se producen con el núcleo o prototipo, tal y como plantea el estudio centrado en las aplicaciones de la disponibilidad léxica a la psicolingüística y a la didáctica (Santos Díaz, Trigo y Romero 2020).

\section{Referencias}

ALBA, Orlando. 1995. Léxico disponible de la República Dominicana. Pontificia Universidad Católica Madre y Maestra.

ÁVILA, Antonio Manuel. 2016. El léxico disponible y la enseñanza del español. Propuesta de selección léxica basada en la teoría de los conjuntos difusos. Journal of Spanish Language Teaching, 3: 31-43.

ÁVILA, Antonio Manuel y Juan Andrés VILLENA(Eds.). 2010. Variación social del léxico disponible en la ciudad de Málaga. Málaga: Editorial Sarriá.

AYORA, María del Carmen. 2006. Disponibilidad léxica en Ceuta: aspectos sociolingüísticos. Cádiz: Servicio de Publicaciones de la Universidad de Cádiz. 
BARTOL, José Antonio. 2001. Reflexiones sobre la disponibilidad léxica. En: BARTOL, José Antonio, Salvador CRESPO, Carmen FERNÁNDEZ JUNCAL, Carmen PENSADO, Emilio PRIETO y Nieves SÁNCHEZ (Eds.). Nuevas aportaciones al estudio de la lengua española. Investigaciones filológicas. Salamanca: LusoEspañola de Ediciones, p. 221-236.

BARTOL, José Antonio. 2004. Léxico disponible de Soria. Estudio y diccionarios. León: Instituto Castellano y Leonés de la Lengua.

BELLÓN, Juan José. 2011. Léxico disponible de la provincia de Córdoba. Córdoba: Servicio de Publicaciones de la Universidad de Córdoba.

CASTILLO FADIĆ, María Natalia y Josué David PINO CASTILLO. 2020. Hacia la construcción de un instrumento para evaluar la familiaridad de pacientes crónicos con unidades léxicas relevantes para el automanejo de su condición de salud. Nueva Revista del Pacífico, 72: 86-115.

CARCEDO, Alberto. 2000. Disponibilidad léxica en español como lengua extranjera: el caso finlandés (estudio del nivel preuniversitario y cotejo con tres fases de adquisición). Turku: Turun Yliopisto.

DEL BARRIO, Florencio. 2018. Pares léxicos en el léxico disponible de estudiantes italianos. Lingue e Linguaggi, 26: 173-196.

DE LA MAYA, Guadalupe. 2015. El desarrollo de la competencia léxica en la adquisición del francés como segunda lengua extranjera en la ESO. Universidad de Extremadura: Tesis doctoral inédita.

DÖRNYEI, Zoltán y Kata CSIZÉR. 2012. How to design and analyze surveys in SLA research? En MACKEY, Alison y Susan GASS (Eds.). Research methods in second language acquisition: A practical guide. London: Wiley-Blackwell p. 74-94.

ECHEVERRÍA, Max Sergio. 1991. Crecimiento de la disponibilidad léxica en estudiantes chilenos de nivel básico y medio. En: LÓPEZ MORALES, Humberto (Ed.). La enseñanza del español como lengua materna. Actas del II Seminario Internacional sobre "Aportes de la lingüística a la enseñanza del español como lengua materna”. Puerto Rico: Universidad de Puerto Rico, p. 61-78.

ECHEVERRÍA, Max Sergio, María Oliva HERRERA, Patricio MORENO y Francisco PRADENAS. 1987. Disponibilidad léxica en Educación Media. RLA. Revista de Lingüística Teórica y Aplicada, 25: 55-115.

ECHEVERRÍA, Max Sergio, VARGAS, Roberto, URZÚA, Paula y Roberto FERREIRA. 2008. DispoGrafo. Una nueva herramienta computacional para el análisis de relaciones semánticas en el léxico disponible, RLA. Revista de lingüística teórica y aplicada, 46: 81-91. 
FERNÁNDEZ JUNCAL, Carmen, y Natividad HERNÁNDEZ MUÑOZ. 2019. Disponibilidad léxica y socionomástica. Ogigia. Revista electrónica de estudios hispánicos, 25: 185-210.

FERREIRA, Roberto y Max Sergio ECHEVERRÍA. 2010. Redes semánticas en el léxico disponible de inglés L1 e inglés LE. Onomázein, 21(1): 133-153.

FERREIRA, Roberto, GARRIDO, Jaime I. y GUERRA, Alexia. 2019. Predictors of lexical availability in English as a second language. Onomázein, 46, 18-34. DOI: 10.7764/onomazein.46.03

GALloso, María Victoria. 2003. El léxico de los estudiantes preuniversitarios en el distrito universitario de Salamanca (Ávila, Salamanca y Zamora). Salamanca: Fundación Instituto Castellano y Leonés de la Lengua.

GÓMEZ DEVÍS, María Begoña. 2003. La disponibilidad léxica de los estudiantes preuniversitarios valencianos: reflexión metodológica, análisis sociolingüístico y aplicaciones. Tesis de doctorado: Universidad de Valencia.

GÓMEZ DEVÍS, María Begoña. 2019. A propósito de las redes semánticas en el léxico disponible de escolares de primero de Educación Primaria, Ogigia. Revista electrónica de estudios hispánicos, 25: 165-183.

GONZÁLEZ FERNÁNDEZ, Javier. 2014. Idoneidad de los centros de interés clásicos en los estudios de disponibilidad léxica aplicados al español como lengua extranjera. Revista Nebrija de Lingüística Aplicada, 16: 1-13.

GOUGENHEIM, George, René MICHEA, Paul RIVENC, Paul y Aurelién SAUVAGEOT. 1956. L'élaboration du francais élémentaire. Paris: Didier.

GOUGENHEIM, George, René MICHEA, Paul RIVENC, Paul y Aurelién SAUVAGEOT. 1964. L'élaboration du francais fondamental (I degré). Étude sur l'élaboration d'un vocabulaire et d'une grammaire de base. Paris: Didier.

HERNÁNDEZ MUÑOZ, Natividad. 2004. El léxico disponible de los estudiantes conquenses. Salamanca: Universidad de Salamanca.

HERNÁNDEZ MUÑOZ, Natividad. 2006. Hacia una teoría cognitiva integrada de la disponibilidad léxica. El léxico disponible de los estudiantes castellano-manchegos. Tesis de doctorado: Universidad de Salamanca.

HERNÁNDEZ MUÑOZ, Natividad y Carmela TOMÉ. 2017. Léxico disponible en primera y segunda lengua: bases cognitivas. En: Del Barrio, FLORENCIO (Ed.). Palabras Vocabulario Léxico. La 
lexicología aplicada a la didáctica y a la diacronía, Firenze: Edizioni Ca' Foscari, p. 99-122.

HIDALGO, Matías. 2017. La disponibilidad léxica como método de detección del vocabulario y de su selección en manuales: Aplicación en una muestra de estudiantes sinohablantes de ELE. Tesis de doctorado: Universidad de Jaén.

HIDALGO, Matías. 2019. El perfil léxico del manual de ELE Español Moderno 1 desde la óptica de la disponibilidad léxica: posibles mejoras para la selección del vocabulario, Ogigia. Revista electrónica de estudios hispánicos, 25: 233-257.

LÓPEZ GONZÁLEZ, Antonio María. 2014a. Disponibilidad léxica. Teoría, método y análisis. Lodz: Universidad de Lodz.

LÓPEZ GONZÁLEZ, Antonio María. 2014b. La estructura interna del léxico disponible en español como lengua extranjera (ELE) de los preuniversitarios polacos. Studia Romanica Posnaniensia, 41(1): 45-61.

LUGONES, Ana. 2015. El léxico disponible de los alumnos de secundaria bilingüe (español e inglés) en Salamanca. Tesis de doctorado: Universidad de Salamanca.

MORENO, Francisco, José Enrique MORENO y Antonio GARCÍA. 1995. Cálculo de disponibilidad léxica. El programa LexiDisp. Lingüística, 7: 243-249.

PAREDES, Florentino. 2012. Desarrollos teóricos y metodológicos recientes de los estudios de disponibilidad léxica, Revista Nebrija de Lingüística Aplicada, 11. https://goo.gl/z7vuJE, accedido el 24/07/2019.

PAREDES, Florentino. 2014. A vueltas con la selección de 'centros de interés' en los estudios de disponibilidad léxica: para una propuesta renovadora a propósito de la disponibilidad léxica en ELE. Revista Nebrija de Lingüística Aplicada, 16: 54-59.

PAREDES, Florentino. 2015. Disponibilidad Léxica y enseñanza de ELE: el léxico disponible como fuente curricular y como recurso en el aula. Linred. Monográfico V Jornadas de Lengua y comunicación: 'Léxico, enseñanza y comunicación': 1-32.

RICHARDS, Jack C. 1969. A Psycholinguistic Measure of Vocabulary Selection. International Review of Applied Linguistics in Language Teaching, 8(2): 87-102.

SAMPER HERNÁNDEZ, Marta. 2002. Disponibilidad léxica en alumnos de español como lengua extranjera. Colección Monografías, n. ${ }^{\circ} 4$. Málaga: ASELE. 
SAMPER PADILLA, José Antonio, Juan José BELLÓN y Marta SAMPER HERNÁNDEZ. 2003. El proyecto de estudio de la disponibilidad léxica en español. Pautas y pistas en el análisis del léxico hispano(americano). Madrid: Vervuert Iberoamericana, p. 27-140.

SÁNCHEZ-SAUS, Marta. 2011. Bases semánticas para el estudio de los centros de interés del léxico disponible. Disponibilidad léxica de informantes extranjeros en las universidades andaluzas. Tesis doctoral: Universidad de Cádiz.

SÁNCHEZ-SAUS, Marta. 2013. Apuntes para una caracterización semántica de los centros de interés en los estudios de disponibilidad léxica. Eugenio Coseriu (1921-2002) en los comienzos del siglo XXI. Analecta Malacitana. Anejo 86: 235-252.

SÁNCHEZ-SAUS, Marta. 2016. Léxico disponible de los estudiantes de español como lengua extranjera en las universidades andaluzas. Sevilla: Universidad de Sevilla.

SÁNCHEZ-SAUS, Marta. 2019. Centros de interés y capacidad asociativa de las palabras. Sevilla: Universidad de Sevilla.

SANTOS DÍAZ, Inmaculada Clotilde. 2017. Selección del léxico disponible: propuesta metodológica con fines didácticos. Porta Linguarum, 27: 122-139

SANTOS DÍAZ, Inmaculada Clotilde. 2020. El léxico bilingüe del futuro profesorado. Análisis y pautas para estudios de disponibilidad. Belín: Peter Lang.

SANTOS DÍAZ, Inmaculada Clotilde; TRIGO, Ester y ROMERO, Manuel Francisco. 2020. La activación del léxico disponible y su aplicación a la enseñanza de lenguas. Porta Linguarum. Revista internacional de didáctica de las lenguas extranjeras, 33: 75-93.

TOMÉ, Carmela. 2011. Reflexiones en torno a los centros de interés. En GALlOSO, M. ${ }^{\mathrm{a}}$ V. (Coord.). La investigación de la Lengua y la Literatura en la Onubense. Huelva: Universidad de Huelva, p. 115-159.

TOMÉ, Carmela. 2015. Léxico disponible. Procesamiento y aplicación a la enseñanza de ELE. Tesis de doctorado: Universidad de Salamanca.

TRIGO, Ester. 2011. Dialectología y cultura. El léxico disponible de los preuniversitarios sevillanos. Valencia: Aduana Vieja.

TRIGO, Ester y Manuel Francisco ROMERO. 2011. Extranjerismos en el centro de interés "la ropa" del léxico disponible de los preuniversiatrios sevillanos. Lingüística Española Actual, 33: 101-124.

TRIGO, Ester, Manuel Francisco ROMERO e Inmaculada Clotilde SANTOS DÍAZ. 2018. Elaboración de un corpus cacográfico desde 
la disponibilidad léxica en estudiantes sevillanos. Un análisis para la enseñanza de la lengua. Revista de Lingüistica y Lenguas Aplicadas, 13: 119-131.

TRIGO, Ester, Manuel Francisco ROMERO e Inmaculada Clotilde SANTOS DÍAZ. 2019. Empirical approach from lexical availability to the influence of sociolinguistic factors on mastery of spelling/Aproximación empírica desde la disponibilidad léxica a la influencia de los factores sociolingüísticos en el dominio ortográfico, Cultura y Educación, 31(4): 814-844. DOI: 10.1080/11356405.2019.1659007.

TRIGO, Ester, Manuel Francisco ROMERO e Inmaculada Clotilde SANTOS DÍAZ. 2020. Disponibilidad léxica y dominio de la ortografía: un estudio empírico basado en la influencia de los factores sociales, Onomázein: Revista de lingüística, filología y traducción, 47: 27-45. DOI: 10.7764/onomazein.47.02.

Recebido em: 15/01/2020

Aprovado em: 04/06/2020 